\title{
Mentorship on malaria microscopy diagnostic service in Ethiopia: baseline competency of microscopists and performance of health facilities
}

Bokretsion Gidey ${ }^{1 *}$, Desalegn Nega ${ }^{1}$, Adugna Abera ${ }^{1}$, Abnet Abebe${ }^{1}$, Sindew Mekasha ${ }^{1}$, Geremew Tasew ${ }^{1}$, Mebrahtom Haile², Dereje Dillu², Degu Mehari², Ashenafi Assefa' ${ }^{2}$, Wondimeneh Liknew',

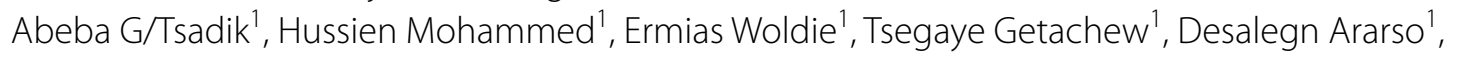
Dereje Yenealem', Adisu Kebede ${ }^{1}$, Kebede Etana², Gizachew Kedida ${ }^{3}$, Hiwot Solomon², Getachew Tollera', Adugna Woyessa ${ }^{1}$ and Ebba Abate ${ }^{1}$

\begin{abstract}
Background: In Ethiopia, malaria cases are declining as a result of proven interventions, and in 2017 the country launched a malaria elimination strategy in targeted settings. Accurate malaria diagnosis and prompt treatment are the key components of the strategy to prevent morbidity and stop the continuation of transmission. However, the quality of microscopic diagnosis in general is deteriorating as malaria burden declines. This study was carried out to evaluate the competency of microscopists and the performance of health facilities on malaria microscopic diagnosis.

Methods: A cross-sectional study was conducted from 1 August to 30 September, 2019 in 9 regional states and one city administration. A standard checklist was used for on-site evaluation, archived patient slides were re-checked and proficiency of microscopists was tested using a WHO-certified set of slides from the national slide bank at the Ethiopian Public Health Institute (EPHI). The strength of agreement, sensitivity, specificity, and positive and negative predictive values were calculated.

Results: In this study, 102 health facilities (84 health centres and 18 hospitals) were included, from which 202 laboratory professionals participated. In slide re-checking, moderate agreement (agreement (A): 76.0\%; Kappa (K): 0.41) was observed between experts and microscopists on malaria detection in all health facilities. The sensitivity and specificity of routine slide reading and the re-checking results were 78.1 and $80.7 \%$, respectively. Likewise, positive predictive value of $65.1 \%$ and negative predictive value of $88.8 \%$ were scored in the routine diagnosis. By panel testing, a substantial overall agreement (A: $91.8 \% ; \mathrm{K}: 0.79)$ was observed between microscopists and experts in detecting malaria parasites. The sensitivity and specificity in the detection of malaria parasites was 92.7 and $89.1 \%$, respectively. In identifying species, a slight agreement (A: 57\%; K: 0.18) was observed between microscopists and experts.
\end{abstract}

Conclusion: The study found significant false positive and false negative results in routine microscopy on slide rechecking of Plasmodium parasites. Moreover, reduced grade in parasite species identification was reported on the

*Correspondence: bokregidey@yahoo.com

${ }^{1}$ Ethiopian Public Health Institute (EPHI), Patriot Street, Gulele Subcity, PO Box 1242, Addis Ababa, Ethiopia

Full list of author information is available at the end of the article

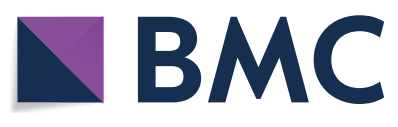

(c) The Author(s) 2021. This article is licensed under a Creative Commons Attribution 4.0 International License, which permits use, sharing, adaptation, distribution and reproduction in any medium or format, as long as you give appropriate credit to the original author(s) and the source, provide a link to the Creative Commons licence, and indicate if changes were made. The images or other third party material in this article are included in the article's Creative Commons licence, unless indicated otherwise in a credit line to the material. If material is not included in the article's Creative Commons licence and your intended use is not permitted by statutory regulation or exceeds the permitted use, you will need to obtain permission directly from the copyright holder. To view a copy of this licence, visit http://creativecommons.org/licenses/by/4.0/. The Creative Commons Public Domain Dedication waiver (http://creativecommons.org/publicdomain/zero/1.0/) applies to the data made available in this article, unless otherwise stated in a credit line to the data. 
panel tests. Implementing comprehensive malaria microscopy mentorship, in-service training and supportive supervision are key strategies to improve the overall performance of health facilities in malaria microscopy.

Keywords: External quality assurance, Mentorship, Malaria microscopy, Re-checking, Competency

\section{Background}

Malaria remains a major public health challenge. In 2019, an estimated 229 million cases of malaria and 409,000 deaths were reported worldwide [1] compared with 238 million cases and 736,000 deaths in 2000, which shows a significant decline. In Ethiopia, around $52 \%$ of the country's population is at risk of the disease. Generally, areas that lay below $2000 \mathrm{~m}$ above sea level are considered malarious. Plasmodium falciparum accounts for nearly $70 \%$ of all malaria cases while the remaining cases are due to Plasmodium vivax, but malaria prevalence is collectively declining from 2011 (4.5\%) to 2015 (1.2\%) [2, 3].

The malaria elimination programme in Ethiopia aims to eliminate malaria through a step-wise and sub-national approach targeting specific adjacent areas in order to shrink the country's malaria map by 2030 [2]. Accurate diagnosis and prompt treatment are core strategies in the elimination of malaria [4]. Diagnosis of malaria is carried out by detecting evidence of parasites or parts of parasites. Microscopic examination of Giemsa-stained blood film is the gold standard for malaria diagnosis [5]. Due to a lack of a sustainable quality assurance programme and trained laboratory technicians, this method has many setbacks in detecting and identifying malaria species correctly in Ethiopia [6-9]. Inaccuracies in diagnostic testing can lead to potentially devastating outcomes for patient and public health, compromising the quality of surveillance data at national level, and ultimately affecting public health policy [10].

Effective malaria diagnosis practice has to be put in place to implement a quality management system that is in line with international quality standards [11]. Strong laboratory capacity with full equipment, reagents and competent professionals ensures better curative interventions and influences treatment-seeking behavior [9] by attracting higher patient flow than facilities with a weak laboratory service. Incorporating a mentoring approach into supervision can transform traditional supervision into a more effective intervention to improve the quality and delivery of patient care [12]. Mentoring typically includes a continued relationship and a broad skills transfer from an individual with more experience in an area to a less experienced mentee in order to improve the performance of laboratory personnel in a health facility [13-16]. An acceptable malaria microscopy service should provide results that are consistently accurate and timely enough to have a direct impact on treatment [15].
This requires comprehensive mentorship and an active quality assurance scheme. This study aimed to evaluate the competency of microscopists and the performance of health facilities on malaria microscopy in Ethiopia. A comprehensive mentorship was provided based on identified gaps.

\section{Methods}

Study area, design and period

A health facility-based, cross-sectional study was conducted from 1 August to 30 September, 2019 at 102 health facility laboratories in 9regional states and one city administration in Ethiopia and the spatial distribution of study sites is depicted in Fig. 1.

\section{Sample size and selection procedure}

In this study, 102 woredas (districts) were selected from each region proportionally according to malaria-reporting health facilities based on annual parasite incidence [17]. The mentor team, in coordination with woreda health offices, selected one health facility per woreda based on their malaria burden report, the presence of medical laboratory professionals and active malaria microscopy diagnostic service.

\section{Expert selection}

Twenty-six experts, 2 from each respective regional Public Health Institute, were selected. Experts were selected based on their experience on malaria microscopy, training on malaria diagnosis and quality assurance, demonstrate ability to transfer knowledge, commitment and willingness to mentor a facility for 3 days with 2 health facilities per week and 8 per month. Five days of standardization training was provided on malaria microscopy and mentorship.

\section{Data collection process}

Thirteen malaria microscopy expert teams, each team with 2 laboratory experts were formed. The experts conducted interviews using a standardized questionnaire using the Open Data Kit (ODK) software programmed on a Smartphone. Panel slides were distributed for proficiency testing. Slides were blind re-checked at health facility level and discordant slides were referred to the national laboratory for confirmation. Real-time data were sent to the Ethiopian Public Health Institute (EPHI) data 


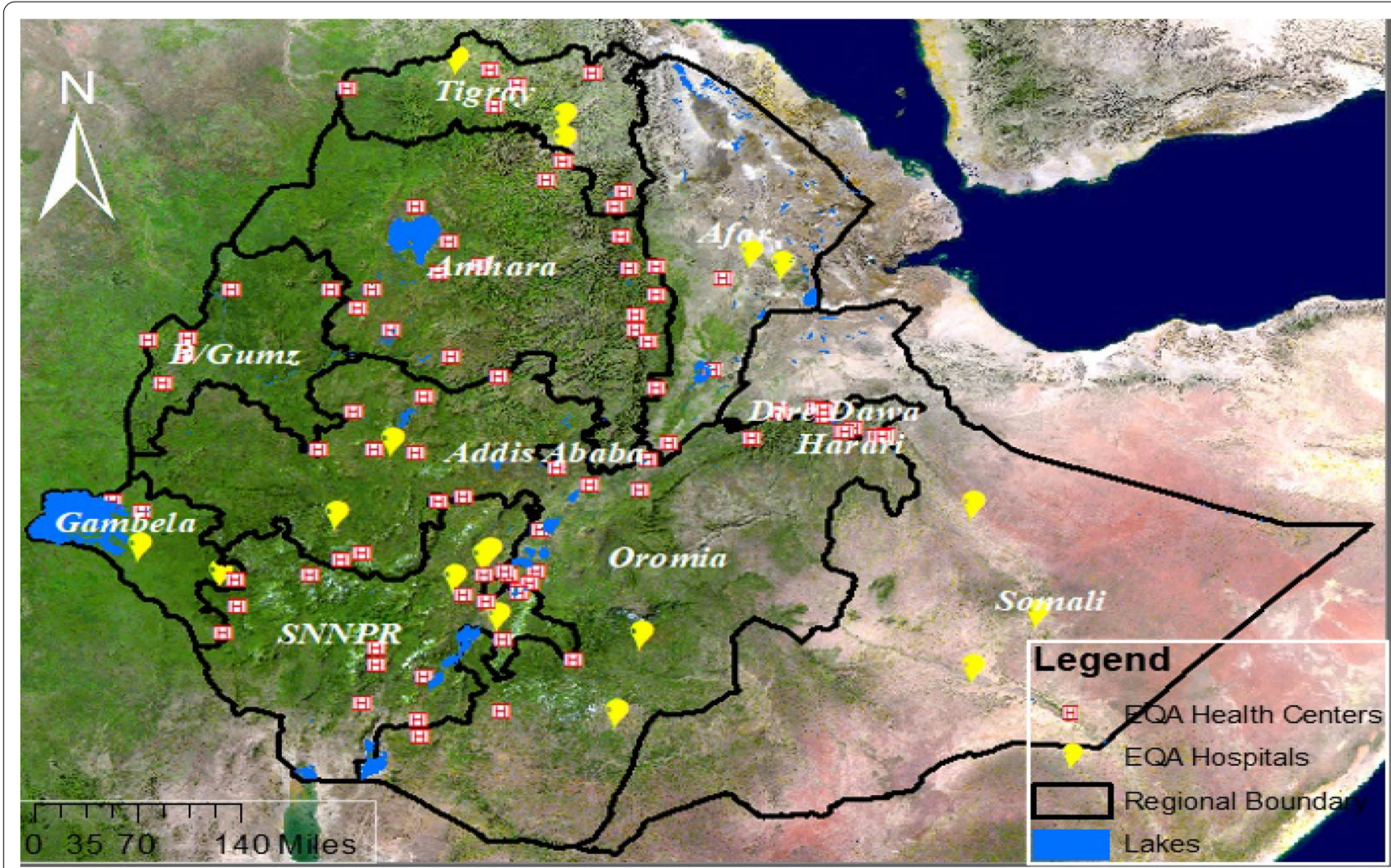

Fig. 1 Spatial distribution of participating health facilities $(\mathrm{N}=102)$ in Ethiopia, 2019

server immediately after data collection completion at each health facility.

\section{On-site evaluation}

A standard supervisory checklist was used to assess the status of documents and records of quality indicators, quality of smear slides, microscopy diagnosis methods and safety practice, equipment and supply management, capacity building issue and training related to malaria microscopy in each health facility.

\section{Proficiency testing}

A set of 4standard reference slides from the national malaria slide bank at EPHI (one slide $P$. falciparum, one $P$. vivax, one mixed (P. falciparum $+P$. vivax) and one negative) were used to test the proficiency of microscopists for parasite detection and species identification.

\section{Slide re-checking}

Expert microscopists (mentors) selected 10stained slides randomly and systematically from the archive of each participant laboratory ( 5 negative and 5 positive stained slides) as per national external quality assurance (EQA) guidelines [9], and assessed health facilities' performance in malaria microscopy diagnosis. Slides were read by experts independently at health facility level and the results were compared immediately.

\section{Data management and statistical analysis}

Real-time data were downloaded from the server, cleaned in Microsoft Excel and imported to SPSS V20 for analysis. Descriptive statistics were used to determine quality monitoring indicators, training, performance of health facilities in malaria microscopy, and competence of laboratory professionals. Sensitivity, specificity, percent agreement, and kappa values were used to determine the competency of laboratory personnel against the expert reader in routine diagnosis. Kappa value was calculated to see the strength of agreement. Strength of agreement was classified as: Kappa of $<0.20$ is slight agreement, 0.21-0.40 fair agreement, $0.41-0.60$ moderate agreement, $0.61-0.80$ substantial agreement, and 0.81-0.99 almost perfect agreement [18].

\section{Ethical consideration}

Ethical approval was obtained from the EPHI Institutional Review Board (IRB) (Protocol number: EPHIIRB-197-2019). An official cooperation letter was written to each health facility. Administrative approvals were 
obtained from the directors of the participating health facilities.

\section{Results}

Demographic characteristics

A total of 102 health facilities were enrolled: 18 (17.6\%) hospitals and $84(82.4 \%)$ health centres from the 9 regional states and one city administration, in Ethiopia. Two-hundred and two laboratory professionals were included as participants in the competence assessment. The median age of the study participants was 29 years (range: $20-55$ years). Most of the participants (68.5\%) were male and 3 in 4 participants, 154 (76.2\%), reported that they had a diploma educational level in medical laboratory technician. Out of the 202 laboratory personnel, $158(78.2 \%)$ were working at health centres. More than half, 107 (53.0\%), of the laboratory personnel had 5 years or more work experience in malaria microscopy (Table 1).

\section{Proficiency testing: detection of malaria parasites and species identification}

Overall performance on detection of parasites

A substantial percent agreement (91.8\%; Kappa 0.79) was observed between study participants and expert references in detecting malaria parasites. The overall sensitivity of malaria parasite detection by participants using microscopy was $92.7 \%$ while the specificity was $89.1 \%$. In the study, $92.7 \%$ positive predictive value and $89.1 \%$ negative predictive values were observed. Almost perfect agreement (92.87\%; Kappa: 0.81) was observed at the health centres and substantial agreement (88.1\%; Kappa: 0.71 ) was observed at hospitals in detecting malaria parasites. The overall score for sensitivity of malaria detection in health centres was $94.5 \%$ which was higher than in hospitals (86.4\%). On the other hand, the specificity of participants in detecting malaria parasites was higher in hospitals (93.2\%) than in health centres (87.9\%) (Table 2).

\section{Overall percent agreement on species identification}

Slight agreement (57\%; Kappa: 0.18) was observed between participants and expert readers in identifying $P$. falciparum from non-P. falciparum parasites. The overall percent agreement in identifying $P$. falciparum and non-P. falciparum was 49\% (Kappa: 0.04) at hospital level and 59\% (Kappa: 0.22) at health centre level. The percent agreement in species identification at health centres was slightly higher than agreement at the hospitals despite low overall agreement at all facilities (Table 3).

\section{Performance of health facilities in malaria microscopy diagnosis \\ Slide re-checking}

Seven-hundred and fifty blood film slides (maximum 10 blood film slides) from each of the 102 assessed health facilities were re-checked by experts. Compared with expert readers, percent agreement of the experts and facilities resulted in malaria parasite detection $76.0 \%$ (Kappa: 0.41) which was at the base boundary of moderate agreement. The sensitivity and specificity were 78.1 and $80.7 \%$, respectively. Similarly, positive and negative

Table 1 Demographic characteristics of health facilities and laboratory personnel, Ethiopia, 2019

\begin{tabular}{|c|c|c|c|}
\hline Characteristics & Variable & Frequency & Percentage (\%) \\
\hline \multirow[t]{2}{*}{ Health facilities enrolled (102) } & Hospitals & 18 & 17.6 \\
\hline & Health centres & 84 & 82.4 \\
\hline \multirow[t]{4}{*}{ Age of laboratory personnel (years) } & $20-30$ & 150 & 74.2 \\
\hline & $31-40$ & 44 & 21.8 \\
\hline & $\geq 41$ & 8 & 4.0 \\
\hline & Total & 202 & 100 \\
\hline \multirow[t]{2}{*}{ Gender } & Male & 139 & 68.8 \\
\hline & Female & 63 & 31.2 \\
\hline \multirow[t]{2}{*}{ Type of college attended } & Government & 121 & 59.9 \\
\hline & Private & 81 & 40.1 \\
\hline \multirow[t]{2}{*}{ Education level } & Diploma & 154 & 76.2 \\
\hline & $\mathrm{BSC}$ & 48 & 23.8 \\
\hline \multirow[t]{3}{*}{ Work Experience (in years) } & $<2$ & 38 & 18.8 \\
\hline & $2-5$ & 57 & 28.2 \\
\hline & $>5$ & 107 & 53.0 \\
\hline \multirow[t]{2}{*}{ Place of work } & Health centre & 158 & 78.2 \\
\hline & Hospital & 44 & 21.8 \\
\hline
\end{tabular}




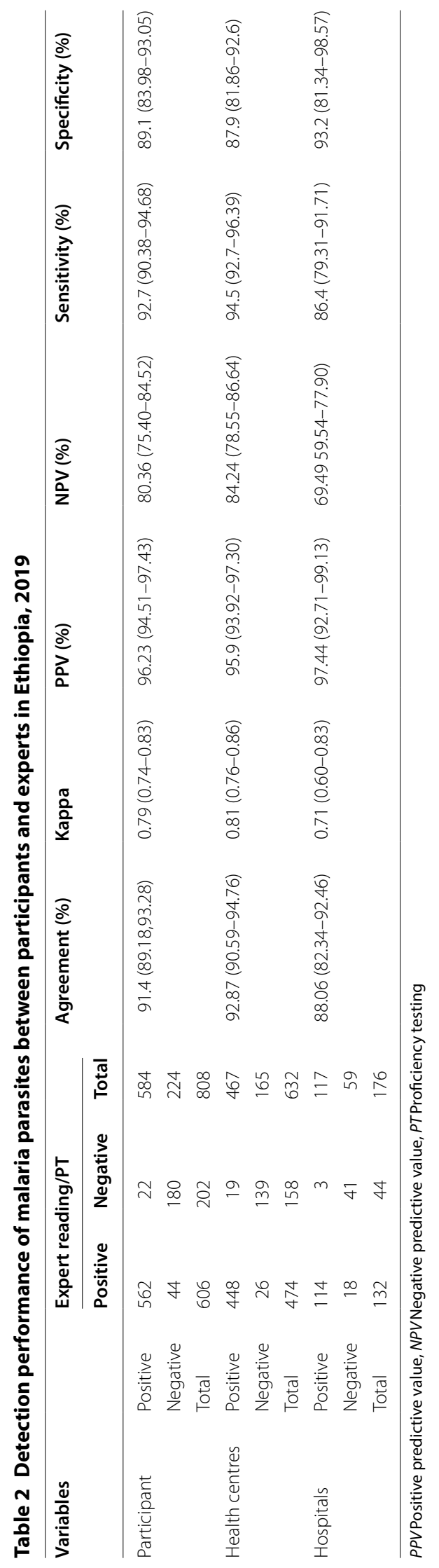


Table 3 Identification performance of Plasmodium falciparum against non-falciparum parasites in Ethiopia, 2019

\begin{tabular}{|c|c|c|c|c|c|c|}
\hline \multirow[t]{2}{*}{ Variables } & & \multicolumn{3}{|l|}{ Expert reading } & \multirow[t]{2}{*}{ Agreement (\%) } & \multirow[t]{2}{*}{ Kappa } \\
\hline & & P. falciparum & $\begin{array}{l}\text { Non-P. } \\
\text { falciparum }\end{array}$ & Total & & \\
\hline \multirow[t]{3}{*}{ Participants' results } & P. falciparum & 147 & 205 & 352 & $57(53.05-61.08)$ & $0.18(0.11-0.26)$ \\
\hline & Non-P. falciparum & 55 & 199 & 254 & & \\
\hline & Total & 202 & 404 & 606 & & \\
\hline \multirow[t]{3}{*}{ Health centre } & P. falciparum & 119 & 154 & 273 & $59(54.71-63.74)$ & $0.22(0.15-0.30)$ \\
\hline & Non- P. falciparum & 39 & 162 & 201 & & \\
\hline & Total & 158 & 316 & 474 & & \\
\hline \multirow[t]{3}{*}{ Hospital } & P. falciparum & 28 & 51 & 79 & $49(40.44-58.08)$ & $0.04(-0.1$ to 0.19$)$ \\
\hline & Non- P. falciparum & 16 & 37 & 53 & & \\
\hline & Total & 44 & 88 & 132 & & \\
\hline
\end{tabular}

predictive values were 65.1 and $88.8 \%$, respectively (Table 4).

\section{On-site evaluation}

Only $72(70.5 \%)$ of the health facilities were performing thick and thin smears on a single slide for every patient. Three in 10 health facilities did not use thin smear for species identification. Less than half the health facilities, $46(45.1 \%)$, monitor the quality of prepared blood film slides regularly. Only one in 3 health facilities, 38 (37.3\%), perform regular internal quality control (IQC) of Giemsa solution and more than 2 in 3 health facilities, 65 (64.7\%), did not store or archive examined slides properly. As part of quality assurance, one of the observed major challenges in most facilities was failure to archive and store examined slides properly, except in 37 (37.3\%) of the health facilities, and only half of $51(50 \%)$ of the health facilities were participating regularly in any method of external quality assurance (Table 5). Sixty-one (59.8\%) health facilities had been supervised by national and/ or regional health offices in the preceding year. Regular training on malaria diagnosis and quality assurance was reported at $26(25.5 \%)$ health facilities. Thirty-two (31.4\%) health facilities diagnosed malaria using both rapid diagnostic tests and microscopy (Table 5).
Box 1 Criteria for assessing quality of malaria blood film slide according to national malaria laboratory diagnosis EQA scheme guideline [9]

Grading Criteria
Excellent Gross appearance: Both thin and thick film prepared on the
same slide, thick film $10 \mathrm{~mm}$ diameter, newsprint read under
thick film before staining, $10 \mathrm{~mm}$ from frosted end and thick
film and between thick and a thin film with distinct head,
body and tail
Microscopic appearances: Demonstrates RBCs lysed in thick
film and a monolayer of RBCs, with normal and abnormal
morphology in thin film. Staining allows the trophozoites,
gametocytes and/or schizonts and the white blood cells to
be clearly distinguished against the background
Gross appearance: Film with uneven tail, too thick, too wide or
too long with uneven thickness
Microscopic appearance: Demonstrates a monolayer of RBCs,
and fixed RBCs
Staining allows the trophozoites, gametocytes and/or schiz-
onts malaria parasites and the white blood cells to be clearly
distinguished against the background
Gross appearance: Film with ragged tail, too thick, too wide or
too long with uneven thickness
Microscopic appearance: Distorted appearance of the RBCs,
malaria parasite and the white cells. It is difficult to spot
fields with monolayer of cells and distorted appearance of
the RBCs, malaria parasite, and the white cells

Table 4 Detection of the malaria parasites in slide re-checking, Ethiopia, 2019

\begin{tabular}{|c|c|c|c|c|c|c|c|c|c|c|}
\hline \multicolumn{2}{|c|}{ Slides from health facilities } & \multicolumn{3}{|c|}{ Expert reading } & \multirow[t]{2}{*}{ Agreement } & \multirow[t]{2}{*}{ Kappa } & \multirow[t]{2}{*}{ Sensitivity } & \multirow[t]{2}{*}{ Specificity } & \multirow[t]{2}{*}{ PPV } & \multirow[t]{2}{*}{ NPV } \\
\hline & & Positive & Negative & Total & & & & & & \\
\hline \multirow[t]{3}{*}{ Health facilities' results } & Positive & 185 & 99 & 284 & $76.0 \%$ & 0.41 & $78.1 \%$ & $80.7 \%$ & $65.1 \%$ & $88.8 \%$ \\
\hline & Negative & 52 & 414 & 466 & & & & & & \\
\hline & Total & 237 & 513 & 750 & & & & & & \\
\hline
\end{tabular}


Table 5 Assessment of quality assurance indicators on malaria microscopy, Ethiopia, 2019

\begin{tabular}{|c|c|c|c|}
\hline Assessment questions & Response & Frequency & Percent (\%) \\
\hline \multirow[t]{3}{*}{ Malaria diagnosis method used } & Microscopy only & 68 & 66.7 \\
\hline & RDTs only & 2 & 1.9 \\
\hline & Both & 32 & 31.4 \\
\hline \multirow[t]{2}{*}{ Prepare both thick and thin films on one slide } & Yes & 72 & 70.5 \\
\hline & No & 30 & 29.5 \\
\hline \multirow[t]{2}{*}{ Monitor the quality of prepared blood film slides } & Yes & 46 & 45.1 \\
\hline & No & 56 & 54.9 \\
\hline \multirow[t]{2}{*}{ Routinely perform IQC in a regular manner } & Yes & 38 & 37.3 \\
\hline & No & 64 & 62.7 \\
\hline \multirow{3}{*}{$\begin{array}{l}\text { Report malaria density or counting malaria parasites in a routine examina- } \\
\text { tion }\end{array}$} & $1^{+}, 2^{+}, 3^{+}, 4^{+}$ & 31 & 30.4 \\
\hline & Parasite/ $\mu$ l & 0 & 0 \\
\hline & Infected RBCs (\%) & 0 & 0 \\
\hline \multirow[t]{2}{*}{ Store and archive examined slides properly } & Yes & 37 & 36.3 \\
\hline & No & 65 & 64.7 \\
\hline \multirow[t]{2}{*}{ Supervised by regional and/or national levels in the last year } & Yes & 61 & 59.8 \\
\hline & No & 41 & 40.2 \\
\hline \multirow[t]{2}{*}{ Obtain refresher training on malaria microscopy diagnosis } & Yes & 26 & 25.5 \\
\hline & No & 76 & 74.5 \\
\hline \multirow[t]{2}{*}{ Participate in any EQA programme regularly } & Yes & 51 & 50 \\
\hline & No & 51 & 50 \\
\hline \multirow[t]{3}{*}{ Quality of prepared and archived slides in the health facilities } & Excellent & 8 & 7.8 \\
\hline & Good & 51 & 50 \\
\hline & Poor & 43 & 42.2 \\
\hline
\end{tabular}

\section{Discussion}

In routine microscopic diagnosis, moderate performance agreement (A) (A: 76.0\%; Kappa: 0.41\%) was observed between study facilities and expert microscopists in parasite detection at all the health facilities. This study showed lower performance agreement compared to a study from the West Amhara Region of Ethiopia [19, 20] and Hawassa, southern Ethiopia [21]. The discrepancy may be due to the large number of health facilities used in the current study. Sensitivity and specificity at detecting malaria in peripheral blood-stained slides were 78.1 and $80.7 \%$, respectively. Similarly, the positive and negative predictive values were 65.1 and $88.8 \%$, respectively. High false positive and false negative results were found at the assessed health facilities, which showed poor performance of their routine diagnosis of malaria. This result was lower than the findings reported from other parts of Ethiopia [19, 20, 22], and similar findings were reported in Pakistan [23]. However, the findings of this study were in line with a study from the Democratic Republic of Congo, which showed performance of routine malaria microscopy remained inaccurate, with large variations among different health centres [24]. Accurate microscopy results depend on the availability of a competent microscopists using good-quality reagents for examining well-prepared slides and with a low-to-moderate workload [25]. In this finding, in slide re-checking, almost half of the assessed health facility laboratories did not prepare both thick and thin blood films as per standard. Consequently, low sensitivity in detection of malaria parasites indicated that there were many false-negative results, i.e., missed diagnosis of true infection. This can lead to delayed treatment, development of serious complications, and death or exposure to unnecessary treatment with other drugs due to suspecting other fever-like diseases.

Regarding proficiency testing, the overall percent agreement of the malaria microscopists in the current study was $91.8 \%$ (Kappa: 0.79) in parasite detection, which is relatively high when compared with similar findings at elimination-targeted districts of Ethiopia where performance agreement was $84.6 \%$ (Kappa: 0.6) [26]. A study conducted in Hawassa town, Ethiopia, reported an agreement of $88 \%$ (Kappa: 0.67) [21] and similar findings were reported from a study conducted in Bahirdar, Ethiopia where agreement was $88.5 \%$ (Kappa: 0.78) [27]. A study conducted in Tigray, Ethiopia, reported an agreement of 79\% (Kappa: 0.62) [28]. A concordant result reported from Addis Ababa public health facilities showed a performance agreement of $91.7 \%$ [29]. But the 
agreement of the current study was relatively low when compared with findings of similar studies conducted in Ethiopia where the agreement was $96.8 \%$ (Kappa: 0.9) [28]. The reason for this deviation may be because of the difference in malaria prevalence which can affect a microscopist's ability to detect, but could also be the lack of mentorship, training, consistent supervision, and capacity building used to develop detecting skills and standardize malaria parasite detection. Overall, sensitivity and specificity of laboratory personnel in detecting malaria parasites were 92.7 and $89.1 \%$, respectively. These results overlapped with positive predictive value $(92.7 \%)$ and negative predictive value (89.1\%). These findings were almost in agreement with a sensitivity $88 \%$ and specificity $91 \%$ from a study conducted in Zambia [30]. The sensitivity but not the specificity of this study was higher than the sensitivity $83.2 \%$ and specificity $90.1 \%$ in a study conducted elsewhere in Ethiopia [26], and sensitivity $63 \%$ and specificity $97 \%$ in a study reported in Tigray, Ethiopia [28]. The sensitivity and specificity of this study was lower than the sensitivity $96.8 \%$ and specificity $96.7 \%$ of malaria detection in a study conducted in Tigray, Ethiopia [28]. The relative lower specificity than sensitivity in the current study at detecting malaria parasites showed that a high rate of false positive results were reported, which led to misdiagnosis of malaria when there was no true infection of malaria parasite in the provided slides.

Overall, the performance agreement on identification of malaria species was 57\% (Kappa: 0.18), which showed a slight agreement between participants and malaria microscopy experts. This result was higher than in a similar study conducted in elimination-targeted districts in Ethiopia with an agreement of 43.8\% (Kappa: 0.11) [26] while it was lower than in a study conducted in Tigray, Ethiopia with an agreement of 76\% (Kappa: 0.61) [28] and a study reported from Bahirdar, Ethiopia where the agreement was $72 \%$ (Kappa: 0.47) [27]. The reason for the low identification of species by microscopists in the current study may be due to the microscopists preparing a thick film only and being unable to differentiate the morphology of the parasites. It may also be due to the lack of training on how to differentiate the species, and to poor staining of reagents found at on-site evaluation.

At on-site evaluation, only $72(70.5 \%)$ health facilities were performing thick and thin smears on a single slide for every patient, but the recommended blood film preparation for diagnosis of malaria parasites is both thin and thick films on the same slide using $2 \mu \mathrm{L}$ and $6 \mu \mathrm{L}$ of whole blood, respectively [31]. Three in 10 health facilities were not using thin smears, which can be used for species identification. This result in the current study is greater than a study conducted in Addis Ababa public health facilities [29]. Moreover, blood films performed in $43(42.2 \%)$ health facilities did not meet the quality of a good blood film for malaria microscopy diagnosis in this study. IQC, used to check the quality of Giemsa stains, was performed only by $38(37.3 \%)$ health facilities. Only $37(37.3 \%)$ of health facilities stored and archived slides properly. Moreover, in the previous year, the study identified in $41(40.2 \%)$ and 76 (74.5\%) health facilities with no supervision and refresher training, respectively. This study was in line with a study conducted in Addis Ababa public health facilities [29] and another study conducted in Asia-Pacific [32]. The results of this study showed that less attention is given to the quality of malaria diagnosis at health facility level. It may be due to lack of refresher training and regular supervision provided to laboratory professionals at health facility level.

\section{Limitations}

There were some limitations where the study health facilities were incorporated purposely based on their high malaria load and presence of microscopic service and the findings are not inferred to occur at all health facilities in the country. The health facilities with no or lower malaria reporting were not included. In addition, a low numbers of slides ( 3 malaria positive and one negative slide) were used in panel testing, which is below WHO standard of 10 slides.

\section{Conclusion}

Most of the malaria microscopists in the current study achieved a good grade agreement in parasite detection. However, a poor grade was obtained in parasite species identification by the panel tests. Moreover, high false positive and false negative results were seen on slide rechecking which showed poor performance of the health facilities in routine malaria microscopy. Poor quality control indicators and follow-up gaps were reported by a significant number of health facilities in this study. An improvement in the quality and accuracy of microscopic diagnosis of malaria is urgently needed. A strong commitment from the National Malaria Elimination Programme and from stakeholders is the vital step to accomplish a mentoring approach at all levels of health facilities.

\section{Abbreviations}

EPHI: Ethiopian Public Health Institute; EQA: External quality assurance; EQAS: External quality assessment; NMEP: National Malaria Elimination Programme; GPS: Global positioning system; IQC: Internal quality control; IRB: Institutional review board; ODK: Open data kit; PT: Proficiency/panel testing; RDTs: Rapid diagnostic tests; SERO: Scientific and ethical review office; PPV: Positive predictive value; NPV: Negative predictive value. 


\section{Acknowledgements}

The authors would like to acknowledge the Ministry of Health for financial support of the field work. We also thank microscopic experts (mentors) from nine regional states and one city administration for providing technical support. Our indisputable appreciation goes to all the malaria microscopists for their willingness to join us for the competence assessment. And more, our especial gratitude goes to Ahlam Awad for English proofreading. Finally, yet importantly, we express our gratitude to the supportive staff of the Ethiopian Public Health Institute who helped in preparing the panels and supplies, facilitated field activities in addressing the logistics and handling financial issues.

\section{Authors' contributions}

All authors contributed in conceptualization, study design, protocol development, training, and field data collection.BG, DN, AdA, AbA, MH, DD, DM, AW, EW analysed the data and wrote the results, BG drafted the manuscript. All authors read and approved the final manuscript.

\section{Funding}

Funding for field data collection and mentoring program was provided by the Ethiopian Federal Ministry of Health.

\section{Availability of data and materials}

The dataset and materials used for the study are kept in a safe place on the EPHI server.

\section{Ethical approval and consent to participants}

The study protocol was reviewed and approved by the Ethiopian Public Health Institute -IRB Office, Addis Ababa, Ethiopia.

\section{Consent for publication}

All authors have read and agreed to publish this article.

\section{Competing interests}

The authors declare that they have no competing interests.

\section{Author details}

${ }^{1}$ Ethiopian Public Health Institute (EPHI), Patriot Street, Gulele Subcity, PO Box 1242, Addis Ababa, Ethiopia. ${ }^{2}$ Federal Ministry of Health (FMoH), Sudan Street, PO Box 80002, Addis Ababa, Ethiopia. ${ }^{3}$ Ethiopian Medical Laboratory Associations (EMLA), Tewodros Square, PO Box: 4866, Addis Ababa, Ethiopia.

Received: 30 September 2020 Accepted: 17 February 2021

Published online: 25 February 2021

\section{References}

1. WHO. World Malaria Report. 20 years of global progress and challenges. Geneva: World Health Organization; 2020. p. 2020.

2. FDRE, Ministry of Health: National Malaria Elimination Roadmap, 2017

3. PMI-Ethiopia: Ethiopia Malaria Operational Plan FY, 2019.

4. FDRE, Ministry of Health: National Malaria Programme Monitoring and Evaluation Plan, 2014-2020. Addis Ababa, Ethiopia, 2014.

5. Maguire JD, Laderman ER, Barcus MJ, Prudhomme WA, Jordon RG, Duong $S$, et al. Production and validation of durable, high quality standardized malaria microscopy slides for teaching, testing and quality assurance during an era of declining diagnostic proficiency. Malar J. 2006:5:92.

6. Carter JY. External quality assessment in resource-limited countries. Biochem Med. 2017:27:97-109.

7. Talapko J, Škrlec I, Alebic T, Jukic M, Včev A. Malaria : the past and the present. Microorganisms. 2019;7:179.

8. FDRE, Ministry of Health: National five-year strategic plan for malaria prevention \& control in Ethioopia 2006-2010. Addis Ababa. 2006

9. FDRE, Ministry of Health: Malaria Laboratory Diagnosis External Quality Assessment Scheme Guidelines. Addis Ababa. 2018.

10. Lippi G, Plebani M, Simundic AM. Quality in laboratory diagnostics : from theory to practice. Biochem Med. 2010;20:126-30.

11. Mathison BA, Pritt BS. Update on malaria diagnostics and test utilization. J Clin Microbiol. 2017:55:2009-17.
12. Aiyenigba B, Ojo A, Aisiri A, Uzim J, Adeusi O, Mwenesi H. Immediate assessment of performance of medical laboratory scientists following a 10-day malaria microscopy training programme in Nigeria. Glob Health Res Policy. 2017;2:32.

13. Perrone AL, Voeurng V, Sek S, Song S, Vong N, Tous C, et al. Implementation research : a mentoring programme to improve laboratory quality in Cambodia. Bull World Health Organ. 2016;94:743-51.

14. Byars-Winston A. A Dahlberg MJ. The National Academies of Sciences: The science of effective mentorship in STEMM. Washington; 2019.

15. Plamondon K, Capacity Building Task Group. Module Three : Why Mentorship ? Canadian Coalition for Global Health Research, 2007.

16. Dee JR, Leišytè L. Organizational learning in higher education institutions: Theories, frameworks, and a potential research agenda. In: Paulsen MB, editor. Higher education: handbook of theory and research. Berlin: Springer Int Publ; 2016. p. 275-348.

17. FDRE, Ministry of Health: National Malaria Programme Report. Addis Ababa, 2017.

18. Viera JA, Garrett MJ. Understanding Interobserver Agreement: The Kappa Statistic. Family Med. 2005;268:2513-4.

19. Hailu AH, Shiferaw BM, Demeke L, Derebe MM, Gelaw DZ, Emiru AM. External quality assessment of malaria microscopy diagnosis among public health facilities in West Amhara Region Ethiopia. BMC Res Notes. 2017; 10:764.

20. Tegegne B, Ejigu K, Alemu G, Feten Y, Endaylalu K, Melese M. Performance of malaria microscopy external quality assessment and networking among health facilities in west Amhara region Ethiopia. BMC Infect Dis. 2020;20:355.

21. Ayalew F, Tilahun B, Taye B. Performance evaluation of laboratory professionals on malaria microscopy in Hawassa. BMC Res Notes. 2014;7:839.

22. Sori G, Zewdie O, Tadele G, Samuel A. External quality assessment of malaria microscopy diagnosis in selected health facilities in Western Oromia. Ethiopia Malar J. 2018;17:233.

23. Khan MA, Walley JD, Munir MA, Khan MA, Khokar NG, Tahir Z, et al. District level external quality assurance (EQA) of malaria microscopy in Pakistan: pilot implementation and feasibility. Malar J. 2011;10:45.

24. Mayengue IP, Batsimba KD, Dossou-Yovo RL, Niama FR, Macosso L, Singana $P B$, et al. Evaluation of routine microscopy performance for malaria diagnosis at three different health centers in Brazzaville Republic of Congo. Malar Res Treat. 2018;2018:4914358.

25. WHO. Malaria microscopy quality assurance manual-Version 2. Geneva: World Health Organization; 2016.

26. Nega D, Abebe A, Abera A, G\Tidey B, Tsadik A, Tasew G. Comprehensive competency assessment of malaria microscopists and laboratory diagnostic service capacity in districts stratified for malaria elimination in Ethiopia. PLoS ONE. 2020;15:0235151.

27. Jemere AK, Melaku YM, Jemeber HT, Abate AM. Performance evaluation of laboratory professionals on malaria microscopy at health facilities in Bahir Dar city administration Northwest Ethiopia. PLOS ONE. 2018;13:e0203420

28. Alemu M, Tadesse D, Hailu T, Mulu W, Derbie A, Hailu T, et al. Performance of laboratory professionals working on malaria microscopy in Tigray North Ethiopia. J Parasitol Res. 2017;2017:9064917.

29. Gebrekidan LD, Hiruy NN. Assessment of malaria microscopic diagnosis performance of laboratory professionals in Addis Ababa's Public Health Facilities. Biomed Sci. 2019;5:1-6.

30. Barat L, Chipipa J, Kolczak M, Sukwa T. Does the availability of blood slide microscopy for malaria at health centers improve the management of persons with fever in Zambia? Am J Trop Med Hyg. 1999;60:1024-30.

31. WHO. Technical consultation to update the WHO Malaria microscopy quality assurance manual. Geneva: World Health Organization; 2014.

32. Ashraf S, Kao A, Hugo C, Christophel EM, Fatunmbi B, Luchavez J, et al. Developing standards for malaria microscopy : external competency assessment for malaria microscopists in the Asia-Pacific. Malar J. 2012;11:352.

\section{Publisher's Note}

Springer Nature remains neutral with regard to jurisdictional claims in published maps and institutional affiliations. 